\title{
Error Performance of Spatial-temporal Combining-based Spatial Multiplexing UWB Systems Using Transmit Antenna Selection
}

\author{
Sangchoon Kim*, Member, KIICE \\ Department of Electronics Engineering, Dong-A University, Busan 604-714, Korea
}

\begin{abstract}
This paper applies transmit antenna selection algorithms to spatial-temporal combining-based spatial multiplexing (SM) ultrawideband (UWB) systems. The employed criterion is based on the largest minimum output signal-to-noise ratio of the multiplexed streams. It is shown via simulations that the bit error rate (BER) performance of the SM UWB systems based on the two-dimensional Rake receiver is significantly improved by antenna diversity through transmit antenna selection on a lognormal multipath fading channel. When the transmit antenna diversity through antenna selection is exploited in the SM UWB systems, the BER performance of the spatial-temporal combining-based zero-forcing (ZF) receiver is also compared with that of the ZF detector followed by the Rake receiver.
\end{abstract}

Index Terms: Ultra-wideband (UWB), Spatial multiplexing, QR decomposition, ZF detector, Rake combiner, Spatialtemporal combining

\section{INTRODUCTION}

Ultra-wideband (UWB) radio technology has attracted tremendous interest for applications requiring high-data rate communications over short ranges. UWB systems trying for higher data rates and higher quality of communications can employ multiple input multiple output (MIMO) techniques [1]. However, the implementation of MIMO suffers the main disadvantage of increasing hardware costs due to the radio frequency (RF) chains and analog-to-digital converters needed. If we use an antenna selection technique, this handicap can be reduced [2-5]. In [6], the transmit antenna selection problem in spatial multiplexing (SM) UWB MIMO systems with zero-forcing (ZF) detectors followed by Rake combiners (called a ZF-Rake), whose structure has been analytically examined in [7], has been discussed. In [8], the 2-dimensional Rake architecture in the space and time domain followed by a ZF detector (called a 2Rake-ZF receiver) has been analytically examined to show that it can improve the error performance of the ZF-Rake receiver.

In this paper, a transmit antenna selection algorithm is applied to the spatial-temporal Rake combining-based SM UWB systems proposed in [8]. The transmit antenna selection criterion employed for selecting a subset of the transmit antennas is based on the largest minimum postprocessing signal-to-noise ratio (SNR) obtained on the basis of QR decomposition. Simulation results will show that the transmit antenna selection algorithm can significantly enhance the BER performance of SM UWB systems with the 2Rake-ZF receiver on a log-normal multipath fading channel. It will also be found that when the transmit antenna

Received 06 July 2012, Revised 27 July 2012, Accepted 03 August 2012

*Corresponding Author E-mail: sckim@dau.ac.kr 
selection scheme is used in the SM UWB systems, the 2Rake-ZF receiver outperforms the ZF-Rake receiver and also achieves the diversity order of the full complexity antenna system.

\section{SPATIAL-TEMPORAL COMBINING SYSTEMS USING TRANSMIT ANTENNA SELECTION}

To examine the antenna diversity gain through transmit antenna selection, the spatial-temporal combining-based SM UWB systems presented in [8] with $N_{T}$ transmit antennas, $N_{R}$ receive antennas, and a $1: N_{S}\left(N_{T}>N_{S}, N_{R} \geq N_{S}\right)$ multiplexer are considered. The subset selection of $N_{S}$ transmit antennas among the set of all possible $N_{\text {search }}=\left(N_{T} ! /\left(N_{S} !\left(N_{T}-N_{S}\right) !\right)\right)$ subsets of transmit antennas is carried out by a transmit antenna selection algorithm on the receiver. The receiver feeds back to the transmitter the determined subset. The input data to the multiplexer are serial-to-parallel converted into $N_{S}$ data streams and then sent to $N_{S}$ transmit antennas for simultaneous transmission, which are modulated with the pulse-amplitude of a UWB pulse with short-duration. The assumption that the pulse repetition interval is adequately larger than the channel delay spread can enable avoiding severe intersymbol interference. It is also assumed that the transmitted signals from the chosen transmit antennas pass through a multipath fading channel with log-normal distribution and the channel coefficients of the $L$ resolvable paths are perfectly known to the receiver.

After the received signal at each receive antenna goes through a correlator, the resulting correlator outputs are spatially and temporally combined by the 2-dimensional Rake receiver over each bit interval, as shown in Fig. 1. Then the discrete-time spatial signal vector, $\mathbf{u}=$ $\left[\begin{array}{llll}u_{1} & u_{2} & \cdots & u_{N_{S}}\end{array}\right]^{T}$, is written as [8],

$$
\mathbf{u}=\sqrt{E_{b}} \mathbf{H}_{p}^{H} \mathbf{H}_{p} \mathbf{b}+\sum_{l=0}^{L-1} \mathbf{H}_{p}(l)^{H} \mathbf{w}(l)
$$

where $E_{b}$ is the average bit energy, $\mathbf{b}=\left[\begin{array}{llll}b_{1} & b_{2} & \cdots & b_{N_{S}}\end{array}\right]^{T}$ with the information bit from the $n$th data stream denoted by $b_{n} \in\{+1,-1\} \quad, \quad n=1,2, \cdots, N_{S} \quad$ and $\quad \mathbf{w}(l)=$ $\left[\begin{array}{llll}w_{1}(l) & w_{2}(l) & \cdots & w_{N_{R}}(l)\end{array}\right]^{T}$ with $w_{m}(l), \quad m=1,2, \cdots, N_{R}$, being the zero-mean white Gaussian noise component of a variance of $N_{0} / 2$ at the $m$-th receive antenna. Here, $\mathbf{H}_{p}$ is a channel matrix corresponding to a subset of best antennas selected from the larger set of available antennas and given by

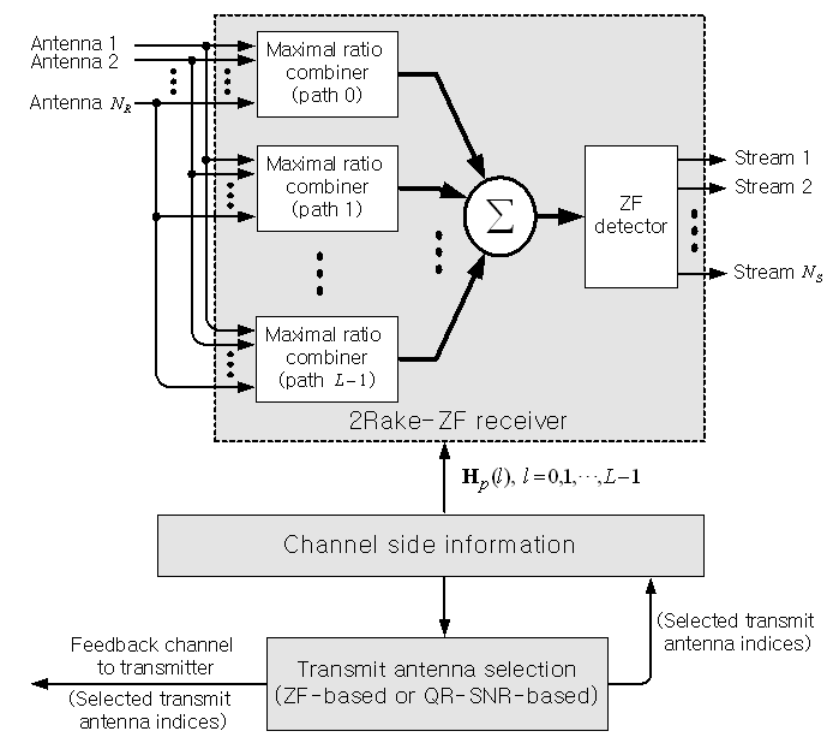

Fig. 1. Block diagram of $2 R$ Rake-ZF receiver with transmit antenna selection. ZF: zero-forcing, SNR: signal-to-noise ratio.

$$
\begin{gathered}
\mathbf{H}_{p}=\left[\begin{array}{llll}
\mathbf{H}_{p}(0)^{T} & \mathbf{H}_{p}(1)^{T} & \cdots & \mathbf{H}_{p}(L-1)^{T}
\end{array}\right]^{T} \\
\mathbf{H}_{p}(l)=\left[\begin{array}{llll}
\mathbf{h}_{1}(l) & \mathbf{h}_{2}(l) & \cdots & \mathbf{h}_{N_{S}}(l)
\end{array}\right] \\
\mathbf{h}_{n}(l)=\left[\begin{array}{llll}
h_{1 n}(l) & h_{2 n}(l) & \cdots & h_{N_{R} n}(l)
\end{array}\right]^{T}
\end{gathered}
$$

where $h_{m n}(l)$ is the channel fading coefficient of the $l$-th resolvable path from the $n$th transmit antenna to the $m$-th receive antenna and can be modeled as $h_{m n}(l)=$ $\zeta_{m n}(l) g_{m n}(l)$ where $g_{m n}(l)$ is the log-normal fading magnitude and $\zeta_{m n}(l) \in\{+1,-1\}$ indicates the phase inversion with equal probability.

After the outputs of the spatial-temporal 2-dimensional Rake combiners are performed by a ZF detector, $\mathbf{G}_{Z F}=\left(\mathbf{H}_{p}^{H} \mathbf{H}_{p}\right)^{\dagger}$, to spatially decorrelate $N_{S}$ data streams, the decision variable is written as,

$$
\begin{aligned}
\mathbf{v} & =\mathbf{G}_{Z F} \mathbf{u} \\
& =\sqrt{E_{b}} \mathbf{b}+\left(\mathbf{H}_{p}^{H} \mathbf{H}_{p}\right)^{\dagger} \mathbf{w}^{\prime}
\end{aligned}
$$

where $(\square)^{\dagger}$ denotes the pseudoinverse and

$$
\mathbf{w}^{\prime}=\sum_{l=0}^{L-1} \mathbf{H}_{p}(l)^{H} \mathbf{w}(l)
$$

Note that this work focuses on using ZF detection even if other SM MIMO detection techniques could be considered. 


\section{TRANSMIT ANTENNA SELECTION FOR SPATIAL-TEMPORAL COMBINING SYSTEMS}

From expression (5), the SNR of the ZF detector output signal on the $k$-th stream in the 2 Rake-ZF receiver using the 2-dimensional combining method in the space and time domain described in Section II can be described as,

$$
S N R_{k}^{(Z F)}=\frac{2 E_{b}}{N_{0}\left[\mathbf{H}_{p}^{H} \mathbf{H}_{p}\right]_{k k}^{-1}}
$$

In order to derive the post-processing SNR expression in the 2Rake-ZF receiver, the channel matrix, $\tilde{\mathbf{H}}_{p}$ $\left(=\mathbf{H}_{p}^{H} \mathbf{H}_{p}\right)$, obtained after spatial-temporal combining is considered instead of using only $\mathbf{H}_{p}$. The $N_{S} \times N_{S}$ modified channel matrix $\tilde{\mathbf{H}}_{p}$ determined by the transmit antenna selection process can be factorized by QR decomposition $\tilde{\mathbf{H}}_{p}=\tilde{\mathbf{Q}}_{p} \tilde{\mathbf{R}}_{p}$ with unitary $N_{S} \times N_{\mathcal{S}}$ matrix $\tilde{\mathbf{Q}}_{p}$ and the upper triangular $N_{S} \times N_{S}$ matrix $\tilde{\mathbf{R}}_{p}$. The left side of the $N_{S} \times 1$ received signal vector $\mathbf{u}$ of the Eq. (1) is multiplied by the Hermitian matrix of $\tilde{\mathbf{Q}}_{p}$. Then the received signal vector is modified to $\mathbf{y}=\tilde{\mathbf{Q}}_{p}^{H} \mathbf{u}$. The postdetection SNR of layer $k$ after spatial and temporal combining can then be given by

$$
S N R_{k}^{(Q R)}=\frac{2 E_{b}}{N_{0}}\left|\tilde{r}_{k, k}\right|^{2}
$$

where $\tilde{r}_{k, k}$ is the $k$-th diagonal element of the upper triangular matrix $\tilde{\mathbf{R}}_{p}$. The elements of $\tilde{\mathbf{R}}_{p}$ can be calculated by the well-known QR factorization algorithm based on a modified Gram-Schmidt method.

An antenna subset selection is determined by the maximum minimum post-detection SNR. It searches for a transmit antenna subset with the maximum value among $N_{\text {search }}$ minimum post-detection SNRs computed for the set of all possible transmit antennas using the expressions (7) and (8). The selection criteria given by the expressions (7) and (8) are called a ZF-based selection criterion (ZF-SC) and a QR-SNR-based selection criterion (QR-SNR-SC), respectively. For every subset of transmit antennas, compute the corresponding $S N R_{\min }^{(Z F)}$ and $S N R_{\min }^{(Q R)}$, respectively, which are defined as minimums of $S N R_{k}^{(Z F)}$, and $S N R_{k}^{(Q R)}$, $k=1,2, \cdots, N_{S}$, using the SNR expressions (7) and (8). Then select the subsets with the maximum $S N R_{\min }^{(Z F)}$ and $S N R_{\min }^{(Q R)}$ values. Finally, the 2 Rake-ZF receiver is operated for signal detection using the determined antenna subset. Thus the 2Rake-ZF receivers based on ZF-SC and QRSNR-SC are called ZF-2Rake-ZF and QR-SNR-2Rake-ZF, respectively.

Even if the communication system with antenna selection considered in [2] and [4] is not a SM UWB one with a lognormal fading distribution, the antenna selection has been shown to maintain the same diversity advantage as the full complexity antenna system without antenna selection. To observe the antenna diversity gain of the ZF-2Rake-ZF and QR-SNR-2Rake-ZF through antenna selection, this work considers the average BER performance of the 2Rake-ZF UWB system with no antenna selection in a log-normal fading channel examined in [8], which is given by

$$
P_{b, 2 \text { Rake-ZF }}=\int_{0}^{\infty} P(t) f(t) d t
$$

where,

$$
\begin{gathered}
P(t)=0.5 \operatorname{erfc}\left(\sqrt{\left(E_{b} / N_{0}\right) t}\right) \\
f(t)=\frac{1}{2^{0.5 D_{2 \text { Rake-ZF }} \Gamma\left(0.5 D_{2 \text { Rake-ZF }}\right)} t^{0.5 D_{2 \text { Rake-ZF }}-1} e^{-0.5 t}}
\end{gathered}
$$

Here, $\quad D_{2 \text { Rake-ZF }}\left(=L N_{R}-N_{T}+1\right)$ indicates the diversity order of the SM UWB system based on the 2Rake-ZF receiver and $\Gamma(\cdot)$ is the gamma function.

\section{SIMULATION RESULTS}

The average power of the path with index $l=0$ is assumed to be unity. The log-normal fading amplitude $g_{m n}(l)$ can be represented by $g_{m n}(l)=e^{\theta_{m n}(l)}$, where $\theta_{m n}(l)$ is a Gaussian random variable with mean $\mu_{\theta_{m n}(l)}$ and variance $\sigma_{\theta}^{2}$ (independent of $l, m$, and $n$ ). It is assumed that the standard deviation of $20 \log _{10} g_{m n}(l)=$ $\theta_{m n}(l)\left(20 \log _{10} e\right)$ is $5 \mathrm{~dB}$. In order to satisfy $E\left[g_{m n}(l)^{2}\right]=e^{-\rho l}$, it is required to be $\mu_{\theta_{m n}(l)}=-\sigma_{\theta}^{2}-\rho l / 2$, where $\sigma_{\theta}$ is obtained as $\sigma_{\theta}=5 /\left(20 \log _{10} e\right)$. Here the power decay factor of $\rho=0$ is used. In the plots, $\left(N_{T}, N_{S}, N_{R}, L\right)$ implies that $N_{T}$ transmit antennas, $N_{S}$ selected transmit antennas, $N_{R}$ receive antennas, and $L$ resolvable paths are used as antenna diversity system parameters. On the other hand, $\left(N_{T}, N_{R}, L\right)$ represents no antenna selection (called No AS) systems.

Fig. 2 shows the BER performance as a function of $E_{b} / N_{0}$ in decibels for the SM UWB $(3,2,2,2)$ antenna selection diversity systems with the 2 Rake-ZF receiver. The antenna diversity performances of the ZF-Rake receiver with ZF-SNR and QR-SNR-based antenna selection criterion (called ZF-ZF-Rake and QR-SNR-ZF-Rake, respectively) are included for comparison. The transmit antenna selection scheme with one more transmit antenna is shown to significantly improve the performance of the 


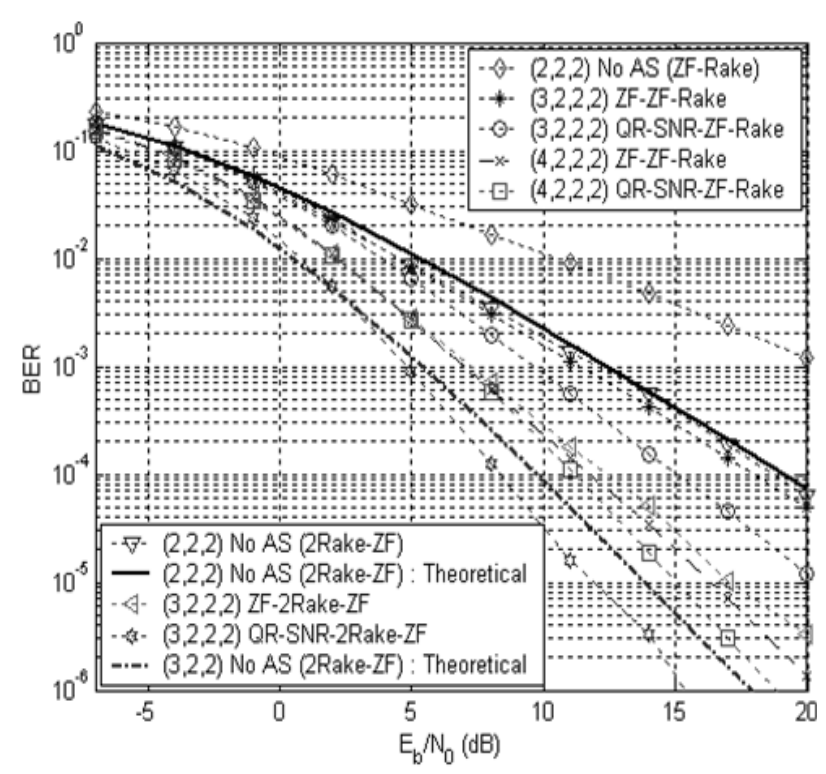

Fig. 2. Bit error rate (BER) versus $E_{b} / N_{0}$ in $(3,2,2,2)$ 2Rake-ZF systems. ZF: zero-forcing, No AS: no antenna selection, SNR: signal-tonoise ratio.

$(2,2,2)$ 2Rake-ZF receiver with no antenna selection. It is also found that the 2 Rake-ZF receiver with antenna selection has much better performance than the ZF-Rake receiver. It is observed that the $(3,2,2,2)$ 2Rake-ZF antenna diversity system outperforms the $(4,2,2,2)$ ZFRake antenna diversity system. It is seen that the QR-SNRSC provides much better performance than the ZF-SC. The BER performance of the $(3,2,2,4)$ 2Rake-ZF system with $L=4$ resolvable multipath components versus $E_{b} / N_{0}$ in decibels is evaluated in Fig. 3 . It is shown that the antenna selection scheme enhances the performance of the 2Rake$\mathrm{ZF}$ receiver even with more resolvable paths, but the degree of performance improvement, which is the performance gap between the antenna selection diversity system and no antenna diversity system, is reduced.

Using expression (9), theoretical BER results of the 2Rake-ZF systems are also plotted in Figs. 2 and 3. It is shown that the $(2,3,2)$ and $(2,3,4)$ 2Rake-ZF MIMO systems can be regarded as full complexity antenna systems without antenna selection of the $(3,2,2,2)$ and $(3,2,2,4)$ 2Rake-ZF antenna selection systems, respectively. The ZF2Rake-ZF has almost the same asymptotic slope as the full system, which means that it can provide the same diversity gain as the full system. Meanwhile, the QR-SNR-2Rake-ZF has a slightly larger slope than the full antenna system, which implies that it offers an additional SNR gain. Thus it is confirmed that the antenna selection achieves the diversity order of the full system.

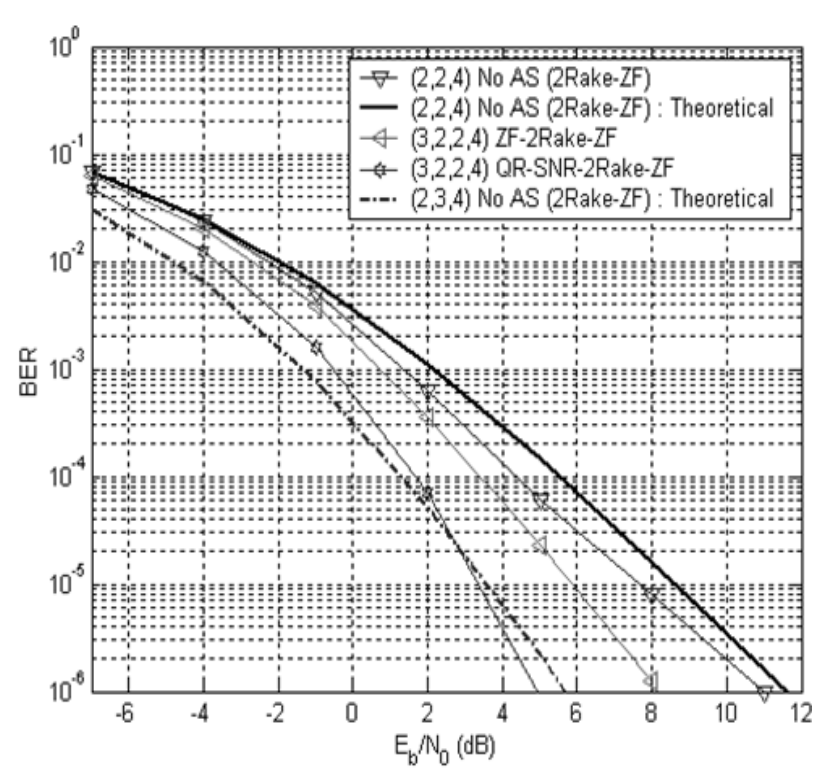

Fig. 3. Bit error rate (BER) versus $E_{b} / N_{0}$ in $(3,2,2,4)$ 2Rake-ZF systems. ZF: zero-forcing, No AS: no antenna selection.

\section{CONCLUSIONS}

The BER performance of a SM UWB system with transmit antenna selection has been evaluated over indoor log-normal fading channels. The employed receiver consists of maximal ratio combiners and multipath combiners that capture $L N_{R}$ resolvable paths in the space and time domain and then a ZF detector to spatially process the $N_{S}$ parallel transmitted data streams. It has been shown that the transmit antenna selection algorithms based on the largest minimum post-detection SNR significantly boost the BER performance of the 2Rake-ZF receiver when the number of multipath combined in time is relatively small. It has been observed that the 2Rake-ZF scheme outperforms the ZF-Rake even with the transmit antenna selection process. It has also been observed that the QR-based antenna selection criterion offers much better performance than the ZF-based selection one. Furthermore, it has been confirmed that the diversity advantage gained by antenna selection is similar to that of the full complexity antenna system.

\section{ACKNOWLEDGMENTS}

This study was supported by research funds from Dong-A University. 


\section{REFERENCES}

[1] T. Kaiser, F. Zheng, and E. Dimitrov, "An overview of ultra-wideband systems with MIMO,” Proceedings of the IEEE, vol. 97, no. 2, pp. 285-312, 2009.

[2] A. F. Molisch and M. Z. Win, "MIMO systems with antenna selection,” IEEE Microwave Magazine, vol. 5, no. 1, pp. 46-56, 2004.

[3] C. Mun, "Transmit-antenna selection for spatial multiplexing with ordered successive interference cancellation,” IEEE Transactions on Communications, vol. 54, no. 3, pp. 423-429, 2006.

[4] R. W. Heath Jr, S. Sandhu, and A. Paulraj, "Antenna selection for spatial multiplexing systems with linear receivers," IEEE Communications Letters, vol. 5, no. 4, pp. 142-144, 2001.
[5] S. H. Tsai, "Equal gain transmission with antenna selection in MIMO communications," IEEE Transactions on Wireless Communications, vol. 10, no. 5, pp. 1470-1479, 2011.

[6] S. Kim, "QR decomposition-based antenna selection for spatial multiplexing UWB systems with zero-forcing detectors followed by rake combiners," IEICE Transactions on Communications, vol. 95B, no. 1, pp. 337-340, 2012.

[7] H. Liu, R. C. Qiu, and Z. Tian, "Error performance of pulse-based ultra-wideband MIMO systems over indoor wireless channels," IEEE Transactions on Wireless Communications, vol. 4, no. 6, pp. 2939-2944, 2005.

[8] J. An and S. Kim, "Spatial-temporal combining-based ZF detection in ultra-wideband communications," IEICE Transactions on Fundamentals of Electronics, Communications and Computer Sciences, vol. 92A, no. 7, pp. 1727-1730, 2009.

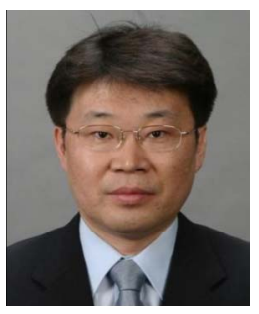

\section{Sangchoon Kim}

received the B.S. degree from Yonsei University, Seoul, Korea, in electronics engineering in 1991 and the M.E. and Ph.D. degrees from the University of Florida, Gainesville, FL, USA, both in electrical and computer engineering, in 1995 and 1999, respectively. From 2000 to 2005, he had been a Research Engineer at the LG Institute of Technology, Seoul and a Senior Research Engineer in LG Electronics, Anyang, Korea. Since 2005, he has been a faculty member in the Department of Electronics Engineering, Dong-A University, Busan, Korea. His research interests include synchronization, array signal processing techniques, and wireless mobile communication systems and algorithms including UWB, MIMO, OFDM, and WCDMA. 